\title{
Inhibition of lactate dehydrogenase A suppresses inflammatory response in RAW 264.7 macrophages
}

\author{
YOO-JEONG SONG ${ }^{1}$, AHYEON KIM ${ }^{1}$, GOON-TAE KIM ${ }^{1}$, HAN YOUNG YU ${ }^{1}$, EUN-SO LEE ${ }^{2}$, \\ MI JIN PARK ${ }^{2}$, YOUNG-JUN KIM ${ }^{3}$, SOON-MI SHIM ${ }^{4}$ and TAE-SIK PARK ${ }^{1}$
}

\author{
${ }^{1}$ Department of Life Science, Gachon University, Sungnam, Gyeonggi 13120; ${ }^{2}$ Department of Dermatology, \\ Ajou University School of Medicine, Suwon, Gyeonggi 16499; ${ }^{3}$ Department of Food and Biotechnology, Korea University, \\ Sejong 30019; ${ }^{4}$ Department of Food Science and Technology, Sejong University, Seoul 05006, Republic of Korea
}

Received March 27, 2018; Accepted September 13, 2018

DOI: $10.3892 / \mathrm{mmr} .2018 .9678$

\begin{abstract}
Lactate is an important metabolite in cellular metabolism and fluctuates in certain disease conditions including cancer and immune diseases. It was hypothesized that a decrease in lactate would modulate the inflammatory response elicited by lipopolysaccharides (LPS) in macrophages. When RAW 264.7 macrophages were treated with FX11, a specific lactate dehydrogenase (LDHA) inhibitor, the expression of the cytokines, inducible nitric oxide synthase (iNOS) and cyclooxygenase 2 (COX-2) was downregulated due to reduced cellular lactate levels. Genetic suppression of LDHA by small interfering RNA (siRNA) downregulated the LPS-activated expression of interleukin (IL)-6, iNOS, and COX-2, and reduced the production of IL- 6 and nitrites. Pharmacological and genetic suppression of LDHA inhibited the phosphorylation of p38 mitogen-activated protein kinase. Microarray gene expression profile demonstrated that the genes involved in cell proliferation and inflammation were mainly altered by siRNA-mediated LDHA suppression. Collectively, the present observations suggest that lactate may be an important metabolite and implicated in regulation of inflammatory response.
\end{abstract}

\section{Introduction}

The immune system distinguishes between self from non-self components, and induces a defensive response against an

Correspondence to: Dr Tae-Sik Park, Department of Life Science, Gachon University, 1342 Sungnamdaero, Sungnam, Gyeonggi 13120, Republic of Korea

E-mail: tspark@gachon.ac.kr

Dr Soon-Mi Shim, Department of Food Science and Technology, Sejong University, 98 Gunja-dong, Gwangjin-gu, Seoul 05006, Republic of Korea

E-mail: soonmishim@sejong.ac.kr

Key words: lactate, inflammation, lactate dehydrogenase, mitogen-activated protein kinase, cytokines invading pathogen; it has evolved to protect the host against pathogenic microbes (1). Tissue injury or infection causes a highly regulated defensive process called inflammation (2), which is a key process in the host defense system. However, excessive inflammation can be a cause for the development of various human diseases including inflammatory disorders, autoimmune disease, cancer, and cardiovascular diseases (3).

Autoimmunity is the loss of immunological self-tolerance in the host organism, which brings about an abnormal immune response against its own self (4). Autoimmune diseases are pathological conditions that lead to chronic inflammation and tissue and organ damage. Among them, neuromyelitis optica spectrum disorder (NMOSD) is caused by demyelination in the central nervous system due to the binding of serum autoantibodies to astrocyte water channel aquaporin-4 (5). Recent metabolomic analyses revealed that lactate levels in cerebrospinal fluid (CSF) from clinical patients with NMOSD are elevated $(6,7)$.

Lactate is the final product resulting from nonoxidative glycolysis, and is considered a waste product of cellular metabolism $(8,9)$. However, abnormal lactate levels have been detected in many diseases such as cancer and rheumatoid arthritis synovitis $(9,10)$. Additionally, lactate has begun to be recognized as an active molecule that regulates the immune response (9). Lactate dehydrogenase is the enzyme that catalyzes the forward and backward conversion of pyruvate to lactate. Lactate dehydrogenase A (LDHA) converts pyruvate to lactate and produces $\mathrm{NAD}^{+}$. On the contrary, lactate dehydrogenase B (LDHB) catabolizes lactate to pyruvate. Previous studies showed that targeting LDHA is an attractive strategy for the development of cancer therapeutics $(11,12)$. In this study, we investigated whether the inhibition of LDHA has anti-inflammatory effects in lipopolysaccharide (LPS)-induced RAW 264.7 macrophages.

\section{Materials and methods}

RAW 264.7 cell culture. Murine RAW 264.7 macrophages were obtained from the American Type Culture Collection (ATCC; Manassas, VA, USA). Cells were cultured in complete high glucose Dulbecco's modified Eagle's Medium (DMEM; Welgene, Inc., Daejeon, Korea) containing 10\% fetal bovine 
serum (FBS), $100 \mathrm{U} / \mathrm{ml}$ penicillin, and $100 \mu \mathrm{g} / \mathrm{ml}$ streptomycin at $37^{\circ} \mathrm{C}$ in a humidified incubator under conditions of $5 \% \mathrm{CO}_{2}$.

Cell viability assay. Cell viability was analyzed by a 3-(4,5-dimethylthiazol-2-yl)-2,5-diphenyltetrazolium bromide (MTT) assay. RAW 264.7 cells were seeded in a 96-well culture plate at a cell density of $2.0 \times 10^{4}$ cells/well. Then, MTT colorimetric solutions were added to each well, and the absorbance was measured at $570 \mathrm{~nm}$ using a microplate spectrophotometer (BioTek instruments, Winooski, VT, USA).

$R N A$ preparation and reverse transcription-quantitative polymerase chain reaction ( $R T-q P C R)$. Total RNA was isolated using the easy-spin Total RNA Extraction kit (iNtRON Biotechnology, Sungnam, Korea), according to the manufacturer's procedure. cDNA was synthesized from RNA in a PCR Thermal Cycler (Takara Bio, Inc., Otsu, Japan) using the iScript cDNA synthesis kit (Bio-Rad Laboratories, Inc., Hercules, CA, USA). RT-qPCR analysis was performed with the SYBR Green Master Mix (Takara Bio, Inc.), using gene-specific primers, in a StepOnelus Real-time PCR system (Applied Biosystems; Thermo Fisher Scientific, Inc., Waltham, MA, USA). PCR conditions were initially maintained at $95^{\circ} \mathrm{C}$ for $5 \mathrm{~min}$, followed by 40 cycles of $95^{\circ} \mathrm{C}$ for $15 \mathrm{sec}, 60^{\circ} \mathrm{C}$ for $15 \mathrm{sec}$ and $72^{\circ} \mathrm{C}$ for $45 \mathrm{sec}$. The level of mRNA expression was normalized to the level of GAPDH expression. The relative value of gene expression was analyzed using the $2^{-\Delta \Delta C \mathrm{C}}$ method (13). The primer sequences used in this study are presented in Table I.

Measurement of lactate in culture media. RAW 264.7 cells were seeded in a 6-well culture plate in the presence and absence of FX11 or LPS. Culture medium was collected and lactate levels were measured by UPLC-tandem mass spectrometry (Triple Quad 5500; Sciex, Framingham, MA, USA), as described previously, with modifications (14).

Western blot analyses. RAW 264.7 cells were seeded in a 6-well culture plate. The next day, cells were treated with different concentrations of FX11 or LDHA-specific small interfering RNA (siRNA) $(50 \mathrm{nM})$. The cells were harvested by lysis buffer (150 mM Sodium chloride, $1 \%$ Triton X-100, $1 \%$ sodium deoxycholate, $0.1 \%$ SDS, $50 \mathrm{mM}$ Tris-HCl, $\mathrm{pH} 7.5$ and $2 \mathrm{mM}$ EDTA) containing protease inhibitors (Roche Diagnostics, Indianapolis, IN, USA) to obtain whole cell lysates. Protein concentrations were measured by using a BCA protein assay kit (Thermo Fisher Scientific, Inc.). Equal amounts of the proteins were subjected to SDS-PAGE, and then transferred onto a PVDF membrane. The membrane was blocked with $5 \%$ skim milk and incubated with specific primary antibodies against p-JNK, JNK, p-ERK, ERK, p-p38, $\mathrm{p} 38$, inducible nitric oxide synthase (iNOS), cyclooxygenase 2 (COX-2), HO-1, and LDHA (Cell Signaling Technology, Inc., Danvers, MA, USA), and $\beta$-actin (EMD Millipore, Billerica, MA, USA). The blots were then incubated with the horseradish peroxidase (HRP)-linked Goat anti-Rabbit (or Mouse) secondary IgG and IgM (EMD Millipore) at room temperature for $1 \mathrm{~h}$. The signals were detected using the SuperSignal West Pico Chemiluminescent Substrate (Thermo Fisher Scientific, Inc.). A chemiluminescence image analyzer (Vilber Lourmat
Corporation, Torcy, France) was used for the detection of signals.

Quantification of nitrite. The concentrations of nitrite in the supernatant of cell culture media were measured by Griess reagent (Molecular Probes; Thermo Fisher Scientific, Inc.). The cell culture media and Griess reagent were taken in a 96-well plate. This mixture was then incubated for $30 \mathrm{~min}$ at room temperature. The nitrite concentrations were measured by measuring the absorbance at $548 \mathrm{~nm}$ using a microplate spectrophotometer (BioTek Instruments Inc., Winooski, VT, USA).

Determination of the levels of pro-inflammatory cytokines secreted. The concentrations of pro-inflammatory cytokines [tumor necrosis factor (TNF)- $\alpha$, interleukin (IL)-1 $\beta$, and IL-6] in the supernatant of cell culture media were measured using a mouse ELISA kit (Enzo Life Sciences, Ann Arbor, MI, USA), according to the manufacturer's instructions.

Transfection of lactate dehydrogenase A-specific siRNA.LDHA silencing in RAW 264.7 macrophages was performed by transiently transfecting the cells with mouse LDHA-specific siRNAs. LDHA-siRNA duplexes and scrambled siRNA were purchased from OriGene Technologies (Rockville, MD, USA). RAW 264.7 cells were seeded in a 6-well culture plate at a cell density of $4 \times 10^{5}$ cells/well. The cells were transfected with LDHA-specific siRNA for 48 h using the Lipofectamine 2000 reagent, according to the manufacturer's instructions (Invitrogen; Thermo Fisher Scientific, Inc.).

Affymetrix whole transcript expression array methods. The Affymetrix whole transcript expression array process was performed according to the manufacturer's protocol (Genechip whole transcript plus reagent kit). cDNA was synthesized using the genechip whole transcript amplification kit according to the manufacturer's instructions. The sense cDNA was then fragmented and biotin-labeled with TdT (terminal deoxynucleotidyl transferase) using the Genechip WT terminal labeling kit. Approximately $5.5 \mu \mathrm{g}$ of the labeled DNA target was hybridized to the Affymetrix genechip mouse 2.0 ST array at $45^{\circ} \mathrm{C}$ for $16 \mathrm{~h}$. The hybridized arrays were washed and stained on a geneChip fluidics station 450 and scanned on a GCS3000 scanner (Affymetrix; Thermo Fisher Scientific, Inc.). Signal values were determined by using the Affymetrix ${ }^{\circledR}$ GeneChip ${ }^{\mathrm{TM}}$ command console software.

Raw data preparation and statistical analysis. Raw data were extracted automatically in Affymetrix data extraction protocol using the software provided by Affymetrix genechip Command Console Software (AGCC). After importing the CEL files, the data were summarized and normalized using the robust multi-average (RMA) method implemented in the Affymetrix expression console software (EC). We exported the result with gene level RMA analysis and performed the differentially expressed gene (DEG) analysis. For a DEG set, a hierarchical cluster analysis was performed using complete linkage and Euclidean distance as a measure of similarity. Gene-Enrichment and functional annotation analysis for significant probe lists was performed using gene ontology (www.geneontology.org/) and KEGG (www.genome.jp/kegg/). 
Table I. Primers used for reverse transcription-quantitative polymerase chain reaction.

\begin{tabular}{ll} 
Target gene & \multicolumn{1}{c}{ Primer sequences (5'-3') } \\
\hline GAPDH & F: CAAGGTCATCCATGACAACTTTG \\
R: GGCCATCCACAGTCTTCTGG \\
FN $\alpha$ \\
F: CAGGCGGTGCCTATGTCTC \\
IL-1 $\beta$ & F: GAAATGCCACCTTTTGACAGTG \\
IL-6 & R: TGGATGCTCTCATCAGGACAG \\
& F: TCCAGTTGCCTTCTTGGGAC \\
iNOS & R: GTGTAATAAGCCTCCGACTTG \\
& F:GTTCTCAGCCCAACAATACAAGA \\
COX-2 & R: GTGGACGGGTCGATGTCAC \\
& F: TGAGTACCGCAAACGCTTCTC \\
HO-1 & R: TGGACGAGGTTTTCCACCAG \\
& F: TGAAGGAGGCCACCAAGGAGG \\
LDHA & R: AGAGGTCACCCAGGTAGCGGG \\
& F: TGTCTCCAGCAAAGACTACTGT \\
& R:GACTGTACTTGACAATGTTGGGA
\end{tabular}

$\mathrm{F}$, forward; R, reverse; TNF- $\alpha$, tumor necrosis factor- $\alpha$; IL-, interleukin; iNOS, inducible nitric oxide synthase; COX-2, cyclooxygenase 2; HO-1, heme oxygenase 1; LDHA, lactate dehydrogenase.

All data analysis and visualization of DEGs were performed by using R 3.1.2 (www.r-project.org).

Isolation of human peripheral blood mononuclear cells (PBMCs). Experiments were performed using whole heparinized blood obtained by venopuncture from healthy donors and from patients with Behçet's disease (Healthy controls, $n=4$. Behçet's disease, $n=6$ ). The present study was conducted in accordance with the provisions of the Declaration of Helsinki for the participation of human subjects in research and was approved by the Institutional Review Board of Ajou University Medical Center (IRB no. BMR-GEN-14-463); written informed consent was obtained from all participants. PBMCs were isolated using histopaque-1077 density-centrifugation and were cultured in RPMI-1640 medium supplemented with $10 \%$ FBS, $1 \%$ non-essential amino acids (100 mM), and $1 \%$ L-glutamine $(200 \mathrm{mM})$. The cultures were maintained at $37^{\circ} \mathrm{C}$ in a $5 \% \mathrm{CO}_{2}$ atmosphere with $95 \%$ humidity.

Data analysis and statistics. All the data are expressed as mean \pm standard error mean. Statistical differences among groups were calculated using one-way analysis of variance with a Bonferroni post hoc test. $\mathrm{P}<0.05$ was considered to indicate a statistically significant difference. Statistical analysis was performed with GraphPad prism 6 software (GraphPad Software, Inc., La Jolla, CA, USA).

\section{Results}

Inhibition of lactate dehydrogenase A suppresses expression of cytokines. Lactate is implicated in excessive cell proliferation and autoimmune diseases $(6,11)$. Therefore, we hypothesized that modulation of lactate regulates inflammatory response induced by LPS. We investigated whether the pharmacological inhibition of LDHA affects the transcriptional expression of cytokines. Among various LDH inhibitors, we utilized FX11 [3-dihydroxy-6-methyl-7-(phenylmethyl)-4-propylnaphthalene-1-carboxylic acid], a gossypol derivative, which demonstrates a higher selectivity for the forward reaction of $\mathrm{LDHA}$, than for the reverse reaction of LDHB $(K i=0.05 \mu \mathrm{M}$ for LDHA, $K i=20 \mu \mathrm{M}$ for LDHB) $(15,16)$. First, we measured the cell viability, to determine a tolerable dose range of FX11 for the study. When RAW 264.7 cells were treated with various concentrations of FX11 for $24 \mathrm{~h}$, it was seen that the cell survival was reduced above FX11 concentrations of $2 \mu \mathrm{M}$ (Fig. 1A); we thus decided to treat the cells with FX11 concentrations below $2 \mu \mathrm{M}$. We treated RAW 264.7 cells with LPS to activate the inflammatory response in the presence or absence of FX11. Inhibition of LDHA did not alter the expression of LDHA. However, a slight decrease in LDHA expression was found in samples treated with $0.5 \mu \mathrm{M}$ FX11, regardless of LPS treatment (Fig. 1B). When the cells were treated with LPS, the lactate levels in the media increased, and FX11 treatment reduced the lactate levels to values lower than those observed in the untreated control (Fig. 1C). This suggests that FX11 pharmacologically inhibited LDHA in RAW 264.7 cells. The transcriptional induction of cytokines including $\mathrm{TNF} \alpha$, IL-1 $\beta$, and IL- 6 by LPS was suppressed when the cells were co-treated with FX11 (Fig. 1D-F). The most notable effect was found in IL-6. These results suggest that reduced lactate levels correlate with the suppression of the LPS-induced expression of cytokines in macrophages.

Inhibition of lactate dehydrogenase A downregulates inflammatory genes and inhibits the phosphorylation of p38 MAP kinase. Inflammatory response induced by bacterial infection activates cytokine production and inflammatory mediators such as nitric oxide (NO) and eicosanoids $(17,18)$. Anti-inflammatory effects were indicated by the suppression of iNOS and COX-2 expression, leading to decreased NO and PGE2 production, respectively (19). In contrast, heme oxygenase 1 (HO-1) downregulates the expression of cytokines such as TNF- $\alpha$, IL-1 $\beta$, and IL-6, and reduces NO production. Thus, HO-1 is suggested as an anti-inflammatory mediator (2). To determine the degree of inflammation regulated by lactate, we examined the effects of FX11 on the transcriptional expression of iNOS and COX-2. Inhibition of LDHA by FX11 downregulated the LPS-induced expression of iNOS and COX-2 (Fig. 2A and B). However, the expressions of iNOS, LDHA, and HO-1 proteins were not changed (Fig. 2D), and the production of nitrite (Fig. 2C) and PGE2 was not altered (data not shown). Only COX-2 was downregulated after treatment with $1 \mu \mathrm{M}$ FX11.

Since FX11 transcriptionally altered the expression of iNOS, COX-2, HO-1, and inflammatory cytokines, we investigated whether the anti-inflammatory effects of FX11 inhibit the phosphorylation of MAP kinases in LPS-induced inflammatory response. We found that the phosphorylation of JNK, ERK, and p38 was reduced by FX11 treatment (Fig. 2E). These results suggest that FX11 downregulates the transcriptional expression of iNOS and COX-2, and inhibits the phosphorylation of MAP kinases. 

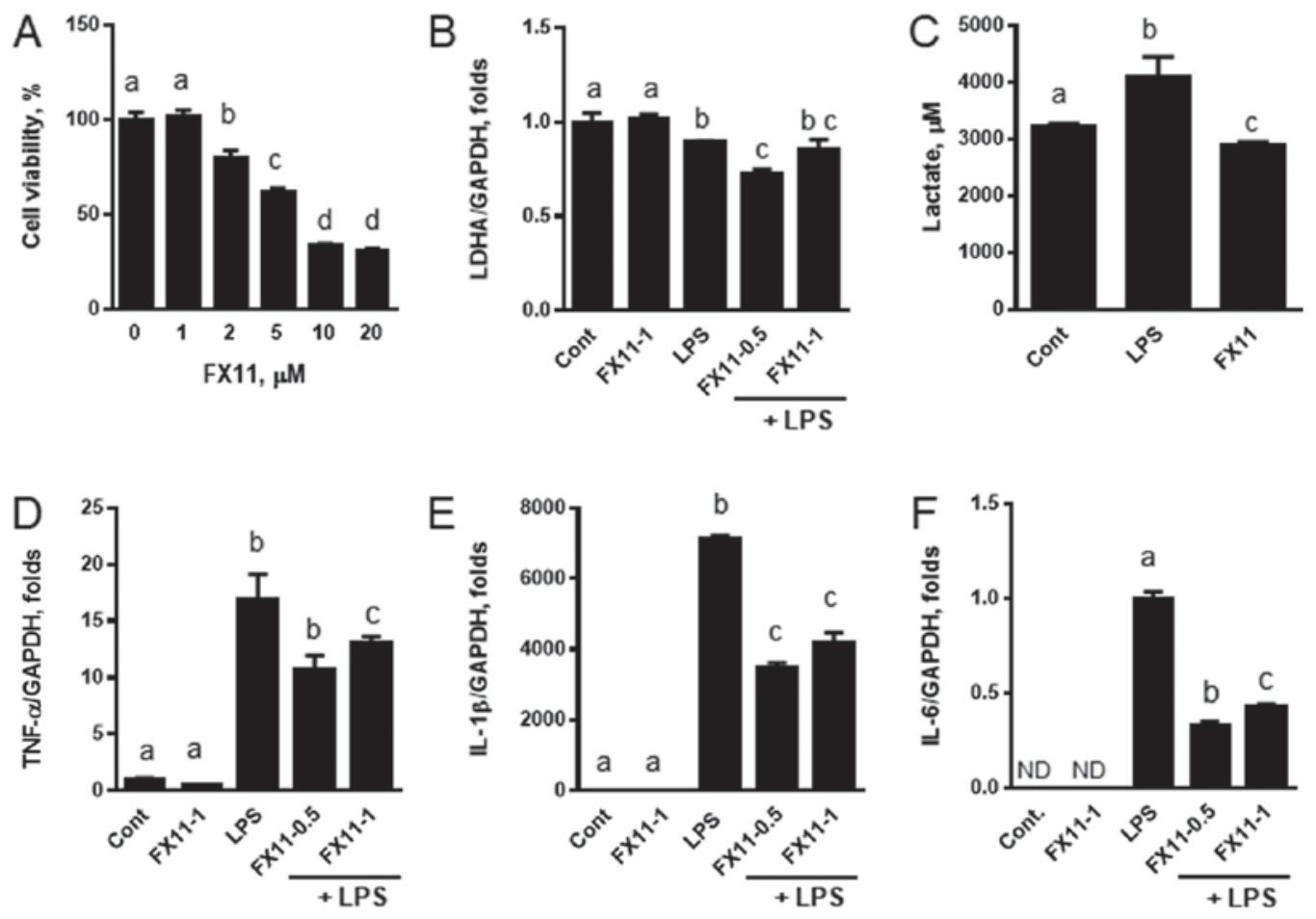

Figure 1. LDHA inhibition by FX11 downregulates the expression of cytokines in LPS-treated RAW 264.7 cells. (A) Raw 264.7 cells were treated with various concentrations of FX11 for $24 \mathrm{~h}$, and the cell viability was measured. (B) LDHA mRNA expression was measured by reverse transcription-quantitative polymerase chain reaction with LPS treatment in the presence or absence of FX11. Numbers following FX11 treatment represent the concentration $(\mu \mathrm{M})$ of FX11 used for treatment. (C) Lactate levels in culture media were measured by ultra-performance liquid chromatography-tandem mass spectrometry. (D) TNF- $\alpha$, (E) IL-1 $\beta$ and (F) IL-6 levels were measured by ELISA. Data are presented as the mean \pm standard error of the mean $(n=3)$. $m R N A$ expression was normalized to the level of GAPDH expression. Different letters indicate a significant difference among treatments at P<0.05. LDHA, lactate dehydrogenase; LPS, lipopolysaccharide; TNF, tumor necrosis factor; IL, interleukin; ND, not detectable.

Suppression of LDHA exerts anti-inflammatory effects via the reduced production of $I L-6$ and nitrites. To support the role of lactate production in anti-inflammatory response, we suppressed LDHA expression by siRNA and examined its effects on the expression of inflammatory genes and signal mediators. LDHA-specific siRNA (siLDHA) downregulated LDHA expression and siLDHA also reduced the LPS-mediated protein induction of iNOS and COX-2 (Fig. 3A). However, the protein levels of HO-1 were not altered. The transcriptional upregulation of IL-6, iNOS, and COX-2 by LPS was attenuated by siLDHA (Fig. 3B-D). Consistent with these expression results, the production of IL-6 and nitrites was reduced when siLDHA was transiently transfected into the cells (Fig. 3E and F). We then examined the effects of genetic downregulation of LDHA on MAP kinase signaling. Although we did not find any changes in the phosphorylation of JNK and ERK, the phosphorylation of $\mathrm{p} 38$ was slightly reduced by siLDHA (Fig. 4A). These results suggest that siLDHA-mediated genetic suppression resulted in anti-inflammatory effects by the downregulation of inflammatory gene expression and inhibition of $\mathrm{p} 38$ phosphorylation.

siRNA-mediated LDHA suppression alters gene expression in transcriptional misregulation in inflammation. Alteration of inflammatory gene expression was found in LDHA-specific siRNA transfected RAW 264.7 cells. To determine the general effects of LDHA suppression, we performed microarray gene analysis using mRNA from both LPS-treated and untreated siLDHA-transfected cells. Gene ontology functional analyses
(Fig. 4B) and pathway analysis (Fig. 4C) revealed that LDHA silencing by siRNA is implicated in diverse signaling pathways. The downregulated genes were clearly enriched in 'cytokine activity' (GO: 0005125), including TNF signaling pathway, chemokine signaling, cytokine-cytokine receptor interaction, and TLR signaling pathway, when we compared cells treated with LPS alone and those treated with LPS plus siLDHA. These results suggest that LDHA knockdown is associated with suppression of cytokine-related inflammatory responses.

We found that the expression of inflammatory and immune-related genes was mainly altered (Fig. 5A). To confirm the involvement of LDHA in inflammation, we measured the expression of CXCL10, which was downregulated as shown in the microarray analyses. We found that pharmacologic or genetic inhibition of LDHA by FX11 treatment or siLDHA transfection, respectively, downregulated CXCL10 in LPS-treated cells (Fig. 5B and C). These results were consistent with those of the microarray analyses. These results suggest that lactate metabolism is positively correlated with inflammatory and immune responses.

Clinical implication of LDHA suppression in PBMCs. To determine the clinical relevance of LDHA inhibition, we treated PBMCs isolated from healthy controls and patients with Behçet's disease with FX-11 in the presence or absence of LPS pre-treatment for $6 \mathrm{~h}$. Behçet's disease is an autoimmune disease characterized by ulceration of the mouth and genitals, inflammation in the eye, and arthritis. IL- $1 \beta$ and TNF $\alpha$ 

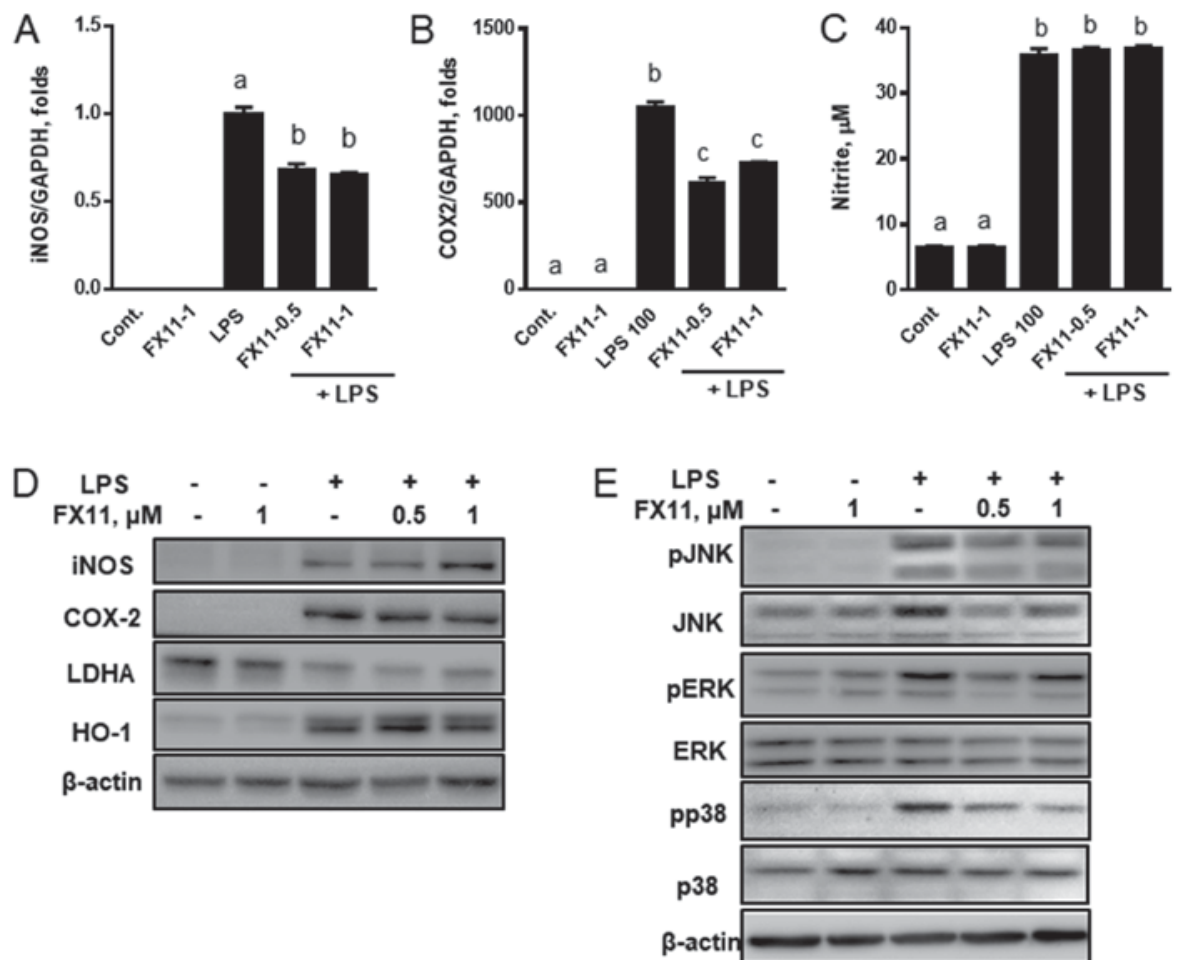

Figure 2. LDHA inhibition by FX11 downregulates the expression of inflammatory genes and inhibits MAP kinase pathways in LPS-treated RAW 264.7 cells. RAW 264.7 cells were treated with LPS in the presence or absence of various concentrations of FX11 ( 0.5 and $1 \mu \mathrm{M})$ for $24 \mathrm{~h}$. The mRNA expressions of (A) iNOS and (B) COX-2 were then measured by reverse transcription-quantitative polymerase chain reaction and the levels of (C) nitrite were measured by Griess reagent assay. (D) Protein levels of iNOS, COX-2, LDHA and HO-1 were measured by immunoblot analyses. (E) The degrees of MAP kinase phosphorylation were measured by immunoblot analyses. Data are presented as the mean \pm standard error of the mean $(\mathrm{n}=3)$. mRNA expression was normalized to the level of GAPDH expression. Different letters indicate a significant difference among treatments at P<0.05. LDHA, lactate dehydrogenase; LPS, lipopolysaccharide; iNOS, nitric oxide synthase; COX-2, cyclooxygenase 2; HO-1, heme oxygenase 1; MAP, mitogen-activated protein; Cont., control; p-, phosphorylated; JNK, c-Jun N terminal kinase; ERK, extracellular signal-regulated kinase.
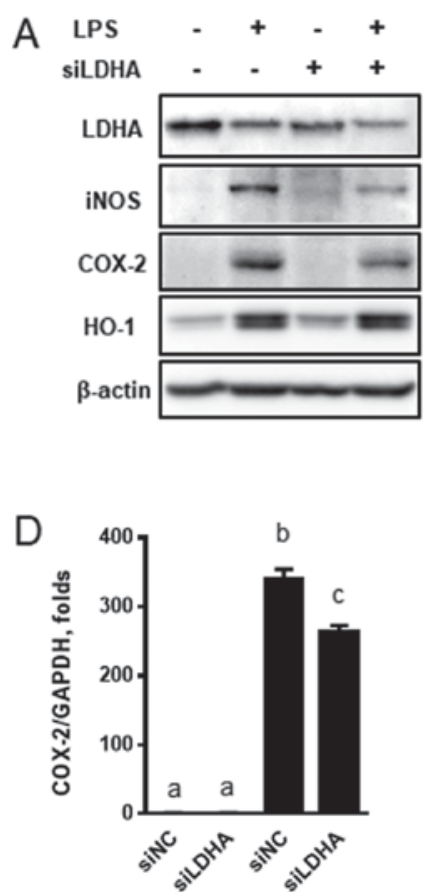

B

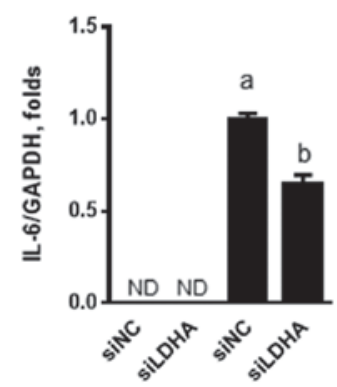

C
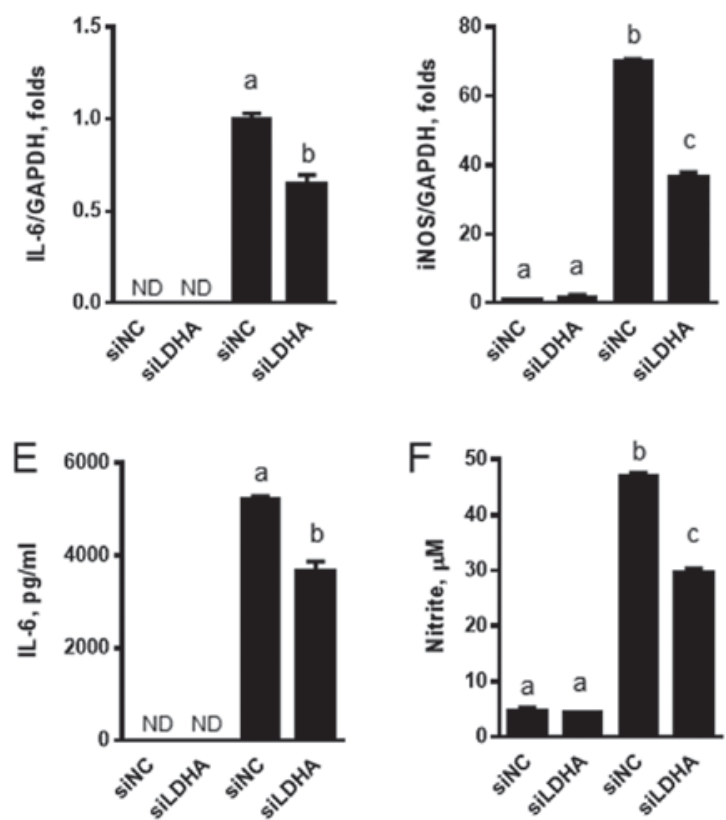

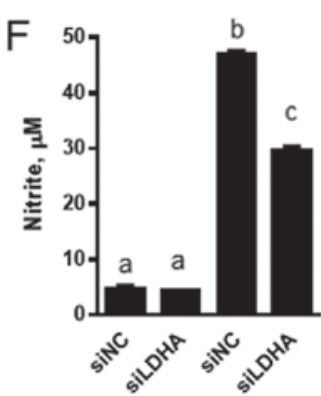

Figure 3. Downregulation of LDHA by siRNA suppresses the expression of inflammatory genes and cytokines. (A) RAW 264.7 cells transfected with LDHA-specific siRNA were treated with LPS in the presence or absence of FX11 for $24 \mathrm{~h}$, and immunoblot analyses were performed with LDHA, iNOS, COX-2, and HO-1. The expressions of (B) IL-6, (C) iNOS and (D) COX-2 were measured by reverse transcription-quantitative polymerase chain reaction. The levels of (E) IL-6 and (F) nitrite were measured by ELISA and Griess reagent respectively. Data are presented as the mean \pm standard error of the mean $(n=3)$. mRNA expression was normalized to the level of GAPDH expression. Different letters indicate a significant difference among treatments at P<0.05. LDHA, lactate dehydrogenase; LPS, lipopolysaccharide; siRNA, small interfering RNA; iNOS, nitric oxide synthase; COX-2, cyclooxygenase 2; HO-1, heme oxygenase 1; ND, not detectable; siNC, scrambled negative control. 

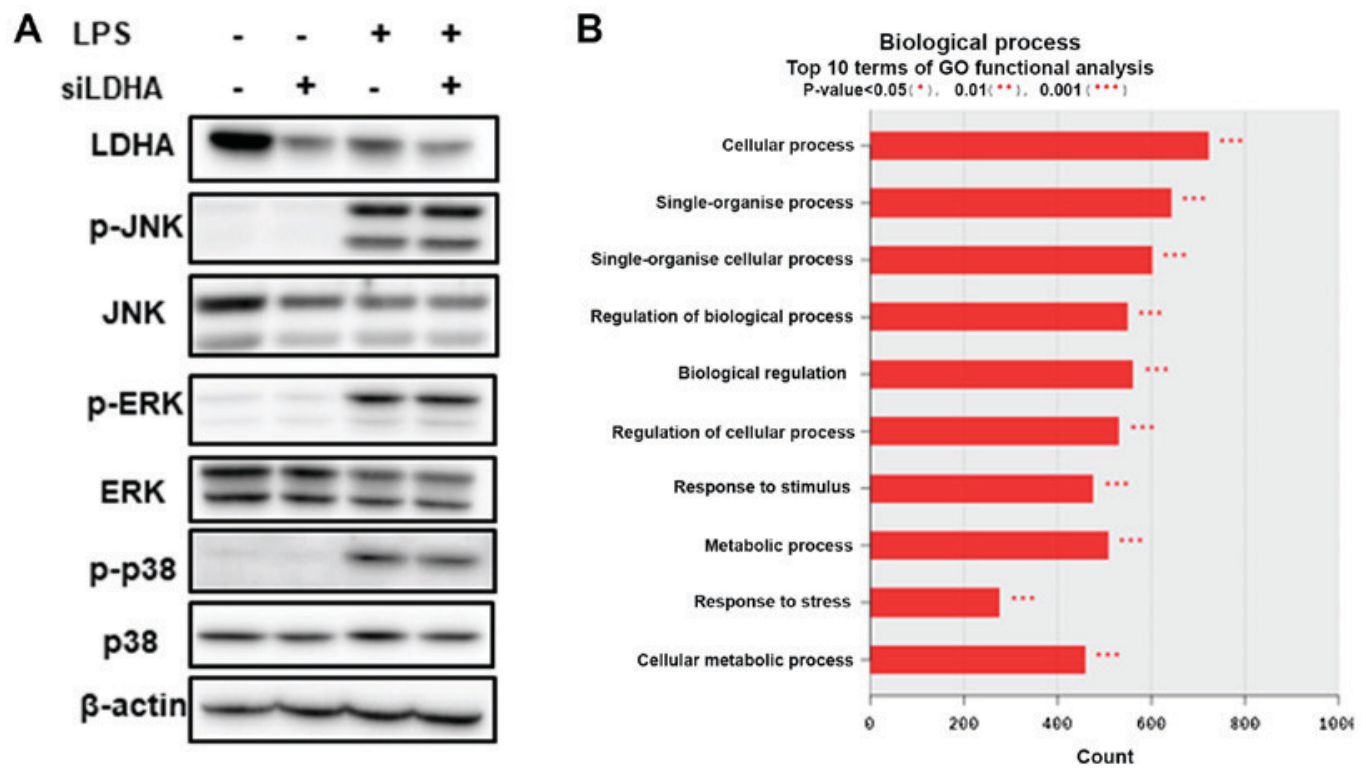

C

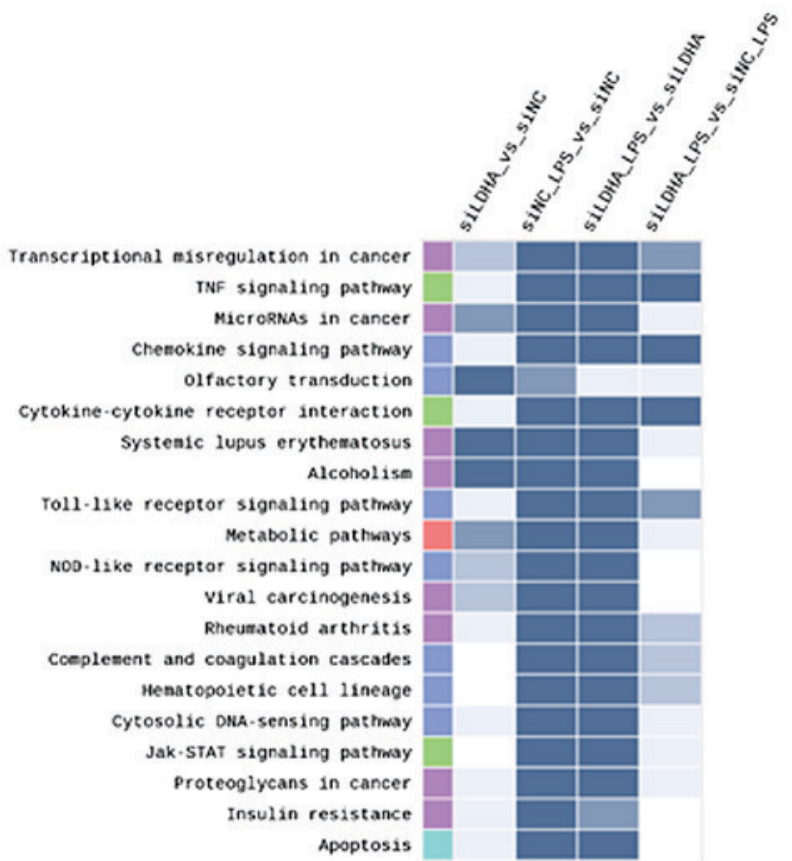

Figure 4. Altered p38 MAP kinase phosphorylation by LDHA silencing and microarray analyses. (A) RAW 264.7 cells transfected with LDHA-specific siRNA were treated with LPS in the presence or absence of FX11 for $24 \mathrm{~h}$, and immunoblot analyses were performed to determine the degree of MAP kinase phosphorylation. The Affymetrix whole transcript expression array process was then performed. (B) GO functional analyses in Biological process and (C) pathway analyses are presented. LDHA, lactate dehydrogenase; LPS, lipopolysaccharide; MAP, mitogen-activated protein; p-, phosphorylated; JNK, c-Jun N terminal kinase; ERK, extracellular signal-regulated kinase; GO, Gene Ontology.

were significantly upregulated by LPS treatment (Fig. 6). When cells were treated with FX11 in the presence of LPS, the expression of IL-1 $\beta$ and TNF $\alpha$ was suppressed in both PBMCs from healthy controls (Fig. 6A) and from patients with Behçet's disease (Fig. 6B). These results suggest that LDHA suppression downregulates the inflammatory response in PBMCs isolated from clinical subjects.

\section{Discussion}

Lactate has long been considered a waste product of cellular metabolism. Currently, lactate is known to serve as an indicator of the status of cell metabolism (20). There have been reports about the correlation of increased lactate levels and development of various diseases including cancer and rheumatoid arthritis synovitis $(9,14)$. Recent reports have demonstrated that the lactate levels in CSF from clinical patients with NMOSD were elevated $(6,7)$. We hypothesized that the suppression of LDHA would downregulate LPS-induced inflammatory response in RAW 264.7 macrophages. To suppress LDHA activity, we administered a pharmacological inhibitor, FX11, and a genetic suppressor, LDHA-specific siRNA. In this study, we demonstrated that: i) The LDHA inhibitor FX11 downregulated the production 
A
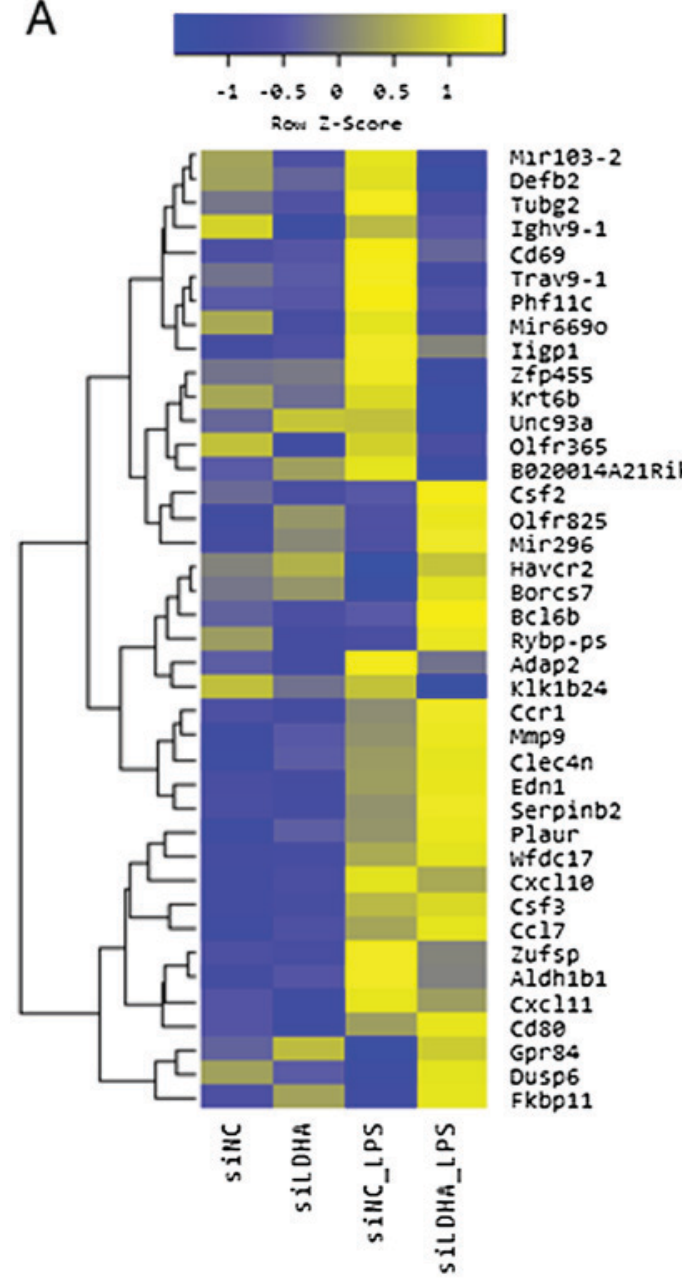

B



C

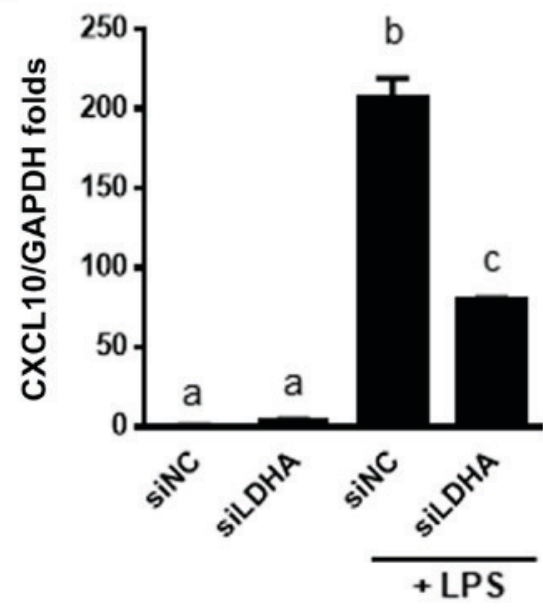

Figure 5. Downregulation of LDHA by siRNA suppresses inflammatory pathways. Affymetrix whole transcript expression array process was performed. (A) Altered representative gene expression with significant change was observed. Among them, the expression of the representative CXCL10 gene in case of treatment with (B) FX11 or (C) LDHA-specific siRNA was measured by reverse transcription-quantitative polymerase chain reaction. Data are presented as the mean \pm standard error of the mean $(n=3)$. mRNA expression was normalized to the level of GAPDH expression. Different letters indicate a significant difference among treatments at $\mathrm{P}<0.05$. LDHA, lactate dehydrogenase; LPS, lipopolysaccharide; siRNA, small interfering RNA; CXCL10, C-X-C motif chemokine ligand 10; siNC, scrambled negative control.

of inflammatory cytokines, iNOS, and COX-2 via the inhibition of MAP kinase phosphorylation; ii) siRNA-mediated suppression of LDHA downregulated the gene expression of iNOS and COX-2, IL-6 production, and NO levels; iii) Suppression of LDHA by LDHA-specific siRNA modulates gene expression of cancer and inflammatory genes, as seen in the results of the microarray analyses.

Lactate is the final product resulting from glycolysis, and is accumulated at inflammatory sites (8). Lactate dehydrogenase catalyzes the conversion of pyruvate to lactate and the reverse reaction. Reports that LDHA inhibition prevents cancer development have drawn attention for the development of cancer therapeutics, and suggest an important role of lactate in the regulation of cell metabolism and survival $(11,21,22)$. Additionally, metabolomics analyses of clinical CSF samples from NMOSD patients suggest that lactate is a critical regulator in the development of autoimmune diseases $(6,7)$. In contrast, the fact that siLDHA-mediated suppression reduced IL-6 and NO production suggests that LDHA inhibition clearly alleviates LPS-mediated inflammatory response. Inconsistency of the results from FX11 and siLDHA treatment could be due to the off-target effects of FX-11. However, the exact reason for this is not clear yet.

Another point is that the phosphorylation of MAP kinases is inhibited by LDHA inhibition. In the immune system, MAP kinases including ERK, JNK, and p38 play an important role in cytokine production, which is a cellular response (23). Cytokines increase the levels of leukocyte adhesion molecules that induce leukocyte extravasation (24). Among the MAP kinase family, ERK is mostly related to anabolic processes including cell division, growth, and differentiation. On the other hand, JNK and p38 are mainly involved in cellular responses in stress condition (25). Some reports reveal that $\mathrm{p} 38$ plays an important role in the production of various inflammatory mediators such as TNF- $\alpha$, IL-1 $\beta$, IL-6, COX-2, and PGE2 (26-28). We expected that the anti-inflammatory effects of LDHA downregulation were associated with the modulation of MAP kinase signaling pathways. Indeed, our studies demonstrated that immunosuppression by FX11-induced LDHA inhibition was closely associated with general MAP kinase signaling pathways. In contrast, LDHA suppression by siRNA only reduced p38 phosphorylation. These results 

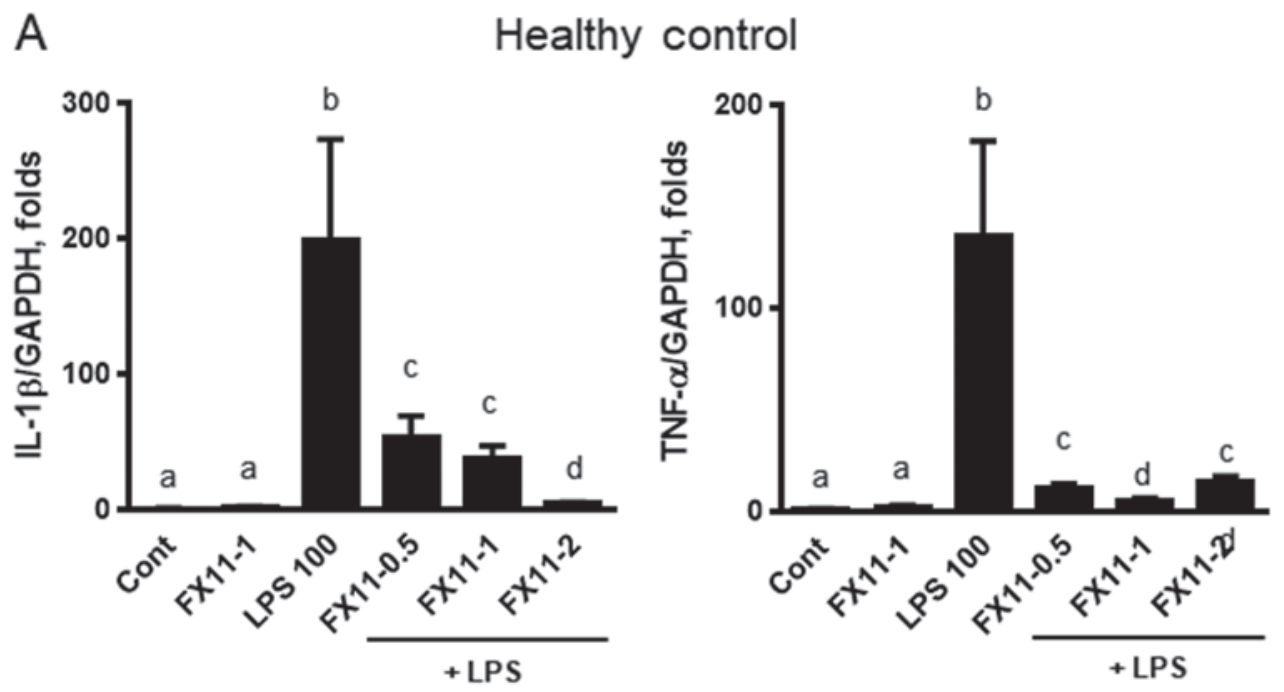

B

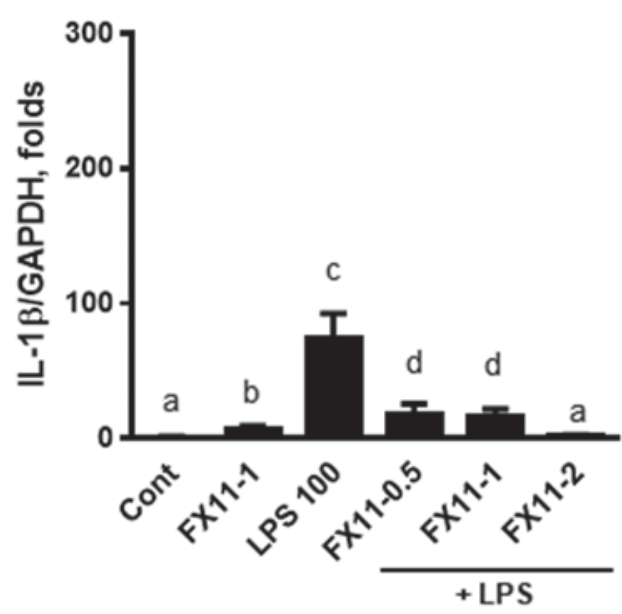

Behçet's disease

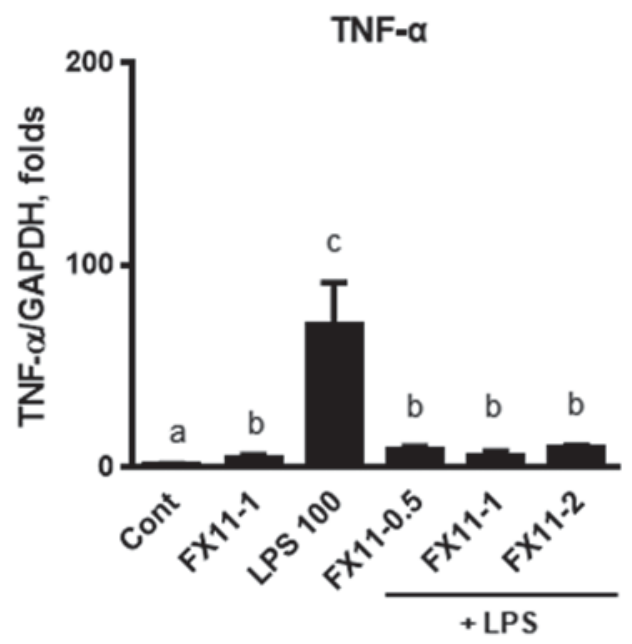

Figure 6. Clinical relevance of lactate dehydrogenase inhibition in PBMCs from human subjects. (A) Healthy controls and (B) patients with Behçet's disease. PBMCs isolated from healthy control human subjects $(n=4)$ and from patients with Behçet's disease $(n=6)$ were pretreated with FX11 at various concentrations for $2 \mathrm{~h}$ prior to treatment with LPS for $4 \mathrm{~h}(100 \mathrm{ng} / \mathrm{ml})$. Expression of IL-1 $\beta$ and TNF- $\alpha$ in PBMCs from the (A) healthy control human subjects and (B) patients with Behçet's disease was measured by reverse transcription-quantitative polymerase chain reaction. Data are presented as the mean \pm standard error of the mean. mRNA expression was normalized to the GAPDH expression level. Different letters indicate a significant difference among treatments at $\mathrm{P}<0.05$. PBMCs, peripheral blood mononuclear cells; LPS, lipopolysaccharide; Cont., control; TNF, tumor necrosis factor; IL, interleukin.

implied that the anti-inflammatory effect of FX11 on phosphorylation of MAP kinases is due to the compound-specific effect. Lactate reduction specifically modulates the p38 MAP kinase pathway, but further studies will be needed to determine the outcome associated with p38 and immunosuppression by LDHA suppression.

Our microarray results suggest that LDHA suppression is associated with various signaling pathways involving 'cytokine activity'. These microarray results suggest that lactate metabolism is positively correlated with inflammatory response. Detailed analysis linking the altered signaling pathways and activation of inflammatory response should be conducted.

The clinical status of autoimmune diseases is that macrophages are already activated, and the secreted inflammatory cytokines exacerbate the inflammatory state. Knowing whether reduction in lactate levels is effective in preventing early activation of macrophages can be critical for applying this strategy in treating autoimmune diseases. The fact that inflammatory cytokine expression was downregulated in PBMCs from healthy control subjects and in patients with autoimmune Behçet's disease upon treatment with LDHA inhibitor suggests that LDHA inhibition is associated with suppression of inflammatory responses in the clinical setting.

Collectively, we found that the suppression of LDHA has anti-inflammatory effects due to the downregulation of several inflammatory mediators including cytokines and NO. Consequently, our findings suggest that LDHA can be a potential therapeutic target for autoimmune diseases via its anti-inflammatory effects in macrophages.

\section{Acknowledgements}

Not applicable. 


\section{Funding}

The present study was supported by the Bio and Medical Technology Development Program through the National Research Foundation of Korea, funded by the Korean government (MSIP; grant no. NRF-2014M3A9B6069338).

\section{Availability of data and materials}

The datasets used and/or analyzed during the current study are available from the corresponding author on reasonable request.

\section{Authors' contributions}

YJS performed the experiments, and prepared the figures and the manuscript. AK, GT and HYY performed the experiments evaluating protein expression. ESL and MJP performed the experiments on clinical samples from patients. YJK and SMS performed virtual screening of the bioactive compounds. TSP conceived and designed the present study, supervised the project and assisted with data interpretation. All authors approved the final manuscript.

\section{Ethics approval and consent to participate}

The present study was conducted in accordance with the provisions of the Declaration of Helsinki for the participation of human subjects in research and was approved by the Institutional Review Board of Ajou University Medical Center (IRB no. BMR-GEN-14-463); written informed consent was obtained from all participants.

\section{Patient consent for publication}

Patient consent for publication was obtained from all participants.

\section{Competing interests}

The authors declare that they have no competing interests.

\section{References}

1. Chaplin DD: Overview of the immune response. J Allergy Clin Immunol 125 (2 Suppl 2): S3-S23, 2010.

2. Jeong YH, Oh YC, Cho WK, Yim NH and Ma JY: Anti-inflammatory effect of rhapontici radix ethanol extract via inhibition of NF-kB and MAPK and induction of HO-1 in macrophages. Mediators Inflamm 2016: 7216912, 2016.

3. Kaminska B: MAPK signalling pathways as molecular targets for anti-inflammatory therapy-from molecular mechanisms to therapeutic benefits. Biochim Biophys Acta 1754: 253-262, 2005.

4. Hewagama A and Richardson B: The genetics and epigenetics of autoimmune diseases. J Autoimmun 33: 3-11, 2009.

5. Alberga D, Trisciuzzi D, Lattanzi G, Bennett JL, Verkman AS, Mangiatordi GF and Nicolotti O: Comparative molecular dynamics study of neuromyelitis optica-immunoglobulin $\mathrm{G}$ binding to aquaporin-4 extracellular domains. Biochim Biophys Acta 1859: 1326-1334, 2017.

6. Park SJ, Jeong IH, Kong BS, Lee JE, Kim KH, Lee DY and Kim HJ: Disease type- and status-specific alteration of CSF metabolome coordinated with clinical parameters in inflammatory demyelinating diseases of CNS. PLoS One 11: e0166277, 2016.
7. Kim HH, Jeong IH, Hyun JS, Kong BS, Kim HJ and Park SJ: Metabolomic profiling of CSF in multiple sclerosis and neuromyelitis optica spectrum disorder by nuclear magnetic resonance. PLoS One 12: e0181758, 2017.

8. Samuvel DJ, Sundararaj KP, Nareika A, Lopes-Virella MF and Huang Y: Lactate boosts TLR4 signaling and NF-kappaB pathway-mediated gene transcription in macrophages via monocarboxylate transporters and MD-2 up-regulation. J Immunol 182: 2476-2484, 2009.

9. Pucino V, Bombardieri M, Pitzalis C and Mauro C: Lactate at the crossroads of metabolism, inflammation, and autoimmunity. Eur J Immunol 47: 14-21, 2017.

10. Hirschhaeuser F, Sattler UG and Mueller-Klieser W: Lactate: A metabolic key player in cancer. Cancer Res 71: 6921-6925, 2011.

11. Le A, Cooper CR, Gouw AM, Dinavahi R, Maitra A, Deck LM, Royer RE, Vander Jagt DL, Semenza GL and Dang CV: Inhibition of lactate dehydrogenase A induces oxidative stress and inhibits tumor progression. Proc Natl Acad Sci USA 107: 2037-2042, 2010.

12. Miao P, Sheng S, Sun X, Liu J and Huang G: Lactate dehydrogenase A in cancer: A promising target for diagnosis and therapy. IUBMB Life 65: 904-910, 2013.

13. Livak KJ and Schmittgen TD: Analysis of relative gene expression data using real-time quantitative PCR and the 2(-Delta Delta C(T)) method. Methods 25: 402-408, 2001.

14. Scheijen JL, Hanssen NM, van de Waarenburg MP, Jonkers DM, Stehouwer CD and Schalkwijk CG: L(+) and D(-) lactate are increased in plasma and urine samples of type 2 diabetes as measured by a simultaneous quantification of $\mathrm{L}(+)$ and $\mathrm{D}(-)$ lactate by reversed-phase liquid chromatography tandem mass spectrometry. Exp Diabetes Res 2012: 234812, 2012.

15. Doherty JR and Cleveland JL: Targeting lactate metabolism for cancer therapeutics. J Clin Invest 123: 3685-3692, 2013.

16. Rellinger EJ, Craig BT, Alvarez AL, Dusek HL, Kim KW, Qiao J and Chung DH: FX11 inhibits aerobic glycolysis and growth of neuroblastoma cells. Surgery 161: 747-752, 2017.

17. Park JY, Pillinger MH and Abramson SB: Prostaglandin E2 synthesis and secretion: The role of PGE2 synthases. Clin Immunol 119: 229-240, 2006.

18. Zhao Y, Vanhoutte PM and Leung SW: Vascular nitric oxide: Beyond eNOS. J Pharmacol Sci 129: 83-94, 2015.

19. Zhou HY, Shin EM, Guo LY, Youn UJ, Bae K, Kang SS, Zou LB and Kim YS: Anti-inflammatory activity of 4-methoxyhonokiol is a function of the inhibition of iNOS and COX-2 expression in RAW 264.7 macrophages via NF-kappaB, JNK and p38 MAPK inactivation. Eur J Pharmacol 586: 340-349, 2008.

20. Andersen LW, Mackenhauer J, Roberts JC, Berg KM, Cocchi MN and Donnino MW: Etiology and therapeutic approach to elevated lactate levels. Mayo Clin Proc 88: 1127-1140, 2013.

21. Fantin VR, St-Pierre J and Leder P: Attenuation of LDH-A expression uncovers a link between glycolysis, mitochondrial physiology, and tumor maintenance. Cancer Cell 9: 425-434, 2006.

22. Xie H, Valera VA, Merino MJ, Amato AM, Signoretti S, Linehan WM, Sukhatme VP and Seth P: LDH-A inhibition, a therapeutic strategy for treatment of hereditary leiomyomatosis and renal cell cancer. Mol Cancer Ther 8: 626-635, 2009.

23. Jeong DH, Kim KB, Kim MJ, Kang BK and Ahn DH: Skipjack tuna (Katsuwonus pelamis) eyeball oil exerts an anti-inflammatory effect by inhibiting NF-kB and MAPK activation in LPS-induced RAW 264.7 cells and croton oil-treated mice. Int Immunopharmacol 40: 50-56, 2016.

24. Newton K and Dixit VM: Signaling in innate immunity and inflammation. Cold Spring Harb Perspect Biol 4: pii: a006049, 2012.

25. Sun J, Ramnath RD, Zhi L, Tamizhselvi R and Bhatia M: Substance P enhances NF-kappaB transactivation and chemokine response in murine macrophages via ERK1/2 and p38 MAPK signaling pathways. Am J Physiol Cell Physiol 294: C1586-C1596, 2008

26. Yang Y, Kim SC, Yu T, Yi YS, Rhee MH, Sung GH, Yoo BC and Cho JY: Functional roles of p38 mitogen-activated protein kinase in macrophage-mediated inflammatory responses. Mediators Inflamm 2014: 352371, 2014.

27. Herlaar E and Brown Z: p38 MAPK signalling cascades in inflammatory disease. Mol Med Today 5: 439-447, 1999.

28. Zarubin T and Han J: Activation and signaling of the p38 MAP kinase pathway. Cell Res 15: 11-18, 2005. 